\title{
La recherche collaborative d'information sur Internet: impact de l'affinité entre les jeunes collaborateurs
}

\author{
JEROME DINET \\ ETIC, Université Paul Verlaine - Metz \\ ROBIN VIVIAN \\ ETIC, Université Paul Verlaine - Metz \\ BRIGITTE SIMONNOT \\ CREM, Université Paul Verlaine - Metz
}

\begin{abstract}
Résumé : Deux études expérimentales visent à décrire les impacts du travail collaboratif et du degré d'affinité entre les collaborateurs lors de recherches d'information sur le Web quand les usagers sont de jeunes apprenants de CM2. Lors de trois sessions successives, les élèves devaient trouver les réponses à des questions liées à l'histoire médiévale en recherchant sur Internet ces réponses. Lors de ces trois sessions, un élève devait effectuer cette recherche d'information seul (condition « Seul »), ou bien avec un ami (condition « Affinité $+»)$, ou bien avec un élève avec lequel il n'entretenait aucun lien amical particulier (condition "Affinité - »). Plusieurs résultats significatifs ont été obtenus : (1) les élèves travaillant en binômes trouvent plus d'informations pertinentes et sont plus efficients que lorsqu'ils travaillent seuls ; (2) les binômes composés d'élèves n'entretenant pas de lien amical trouvent plus d'informations pertinentes et sont plus efficients ; (3) les binômes composés d'élèves entretenant des liens amicaux produisent plus de requêtes non pertinentes; (4) les binômes composés d'élèves n'entretenant pas de lien amical sont plus engagés dans des interactions facilitatrices.
\end{abstract}

Mots clés : Recherche collaborative d'information, Interactions, Aspects affectifs.

\begin{abstract}
Two experimental studies aim at describing the impacts of pair interaction and affective factors during the process of collaborative search for information on the World Wide Web when end-users are young learners recruited from Grade 3. In three successive sessions, students were asked to find answers to questions related to medieval history on the Internet. In these three sessions, students were asked to find these answers alone (condition "Alone"), or with a friend (condition "Affinity +"), or with another pupil who was not a friend (condition "Affinity -"). Several significant results have been obtained: (1) pairs retrieved effectively more answers, more correct answers, and were more efficient than singles; (2) pairs composed of children without social affinity retrieved more answers, more correct answers and were more efficient than pairs composed of friends; (3) pairs composed of friends produced significantly more irrelevant queries than pairs composed of children without social affinity; (4) pairs composed of children without social affinity are engaged in a great deal of consensus seeking, compared with the pairs composed of friends.
\end{abstract}

Key words: Collaborative information search, Interaction, Affective factors.

Les articles de JIPS sont publiés sous licence Creative Commons Paternité 2.0 Générique. 


\section{INTRODUCTION}

Cet article présente les résultats de deux expérimentations s'intéressant aux impacts du degré d'affinité qui lie deux jeunes élèves devant, ensemble et de manière collaborative, rechercher des informations sur Internet. Cet article approfondit et complète l'étude présentée à la conférence IHM'2008 [Dinet, Simonnot and Vivian 2008]. Ces deux expérimentations reposent sur un triple constat :

- La recherche collaborative d'information sur Internet est aujourd'hui une réalité dans de nombreux contextes (école, collège, lycée, université, entreprise, etc.) [Dinet 2007]. Par exemple, à l'école, ce sont le plus souvent des binômes qui doivent réaliser l'activité conjointement pour des raisons pédagogiques et des raisons liées au manque de matériel et de temps ;

- Si certaines études empiriques [Diamadis and Polyzos 2004], [de Vries et al., 2008] tendent à montrer que la recherche collaborative est plus efficace que la recherche conduite individuellement (gain de temps, nombre de sites explorés plus important, diminution des revisites, meilleure organisation des sites visités), les résultats sont d'une part, rarement généralisables à cause d'effectifs trop restreints et d'une méthodologie imprécise et d'autre part, ne concernent que des populations d'étudiants et/ou d'adultes ;

- Si la plupart des auteurs s'intéressant à la recherche collaborative d'information sur Internet reconnaissent le rôle primordial des liens affectifs (amitié, inimitié, indifférence, etc.) entre les collaborateurs [Dumais, Cutrell and Chen 2001] [Hansen and Järvelin 2005], aucune étude à notre connaissance n'a cherché à tester les impacts de ces liens sur les comportements, performances et interactions entre les individus.

Aussi, les trois principales questions auxquelles nous tentons d'apporter des éléments de réponse dans cet article sont les suivantes :

1. La recherche collaborative d'information sur Internet est-elle réellement plus efficace que la recherche individuelle lorsqu'il s'agit de jeunes usagers (élèves de CM2)?

2. Le degré d'affinité entre les membres d'un binôme influence-t-il leurs comportements et stratégies?

3. Quels sont les impacts au niveau des interactions de ce degré d'affinité entre les deux collaborateurs?

Dans un premier temps, nous proposons de définir la recherche collaborative d'information, notamment en proposant une typologie des situations et en nous centrant sur les différences entre la recherche collaborative et la recherche individuelle d'information. Puis, nous présentons les deux expérimentations: la première étude s'intéresse essentiellement aux performances et comportements de jeunes élèves devant rechercher des informations sur Internet, tantôt seuls, tantôt en binômes (dont le degré d'affinité varie) ; la seconde étude se focalise davantage sur les échanges verbaux entre les élèves dans les situations de recherche collaborative, i.e. au sein des binômes.

\section{LA RECHERCHE COLLABORATIVE D'INFORMATION}

\section{Définition}

En accord avec la définition de [Hansen \& Järvelin 2005], nous pouvons définir la recherche collaborative d'information de la manière suivante : 
« est considérée comme recherche collaborative d'information toute tâche de type résolution de problèmes, impliquant plusieurs individus interagissant, de manière synchrone ou asynchrone, lors d'une tâche commune de recherche de sites ou de pages Web dans des contextes plus ou moins définis et des environnements plus ou moins ouverts ».

La recherche collaborative d'information s'intéresse donc aux activités qu'un groupe de personnes entreprennent pour identifier et résoudre un besoin d'information partagé. Mais, cette définition est trop généraliste. En effet, nous pouvons plus finement distinguer les activités de recherche collaborative d'information selon trois facteurs qui interagissent :

(1) le statut des partenaires (collaborateurs) concernés, avec les cas de figures suivants :

- Un utilisateur "novice» tout-venant et un expert. Ce dernier peut être expert dans l'activité (documentaliste, bibliothécaire) ou être expert dans le domaine concerné par la recherche (informaticien, chirurgien, pilote aéronautique) ;

- Plusieurs utilisateurs novices (tels que des élèves);

- Plusieurs experts ;

(2) le type de média partagé par les collaborateurs pour réaliser l'activité :

- Sans média technique. Dans ce cas, les collaborateurs interagissent dans des situations de face-à-face, par les canaux verbaux ou paraverbaux (gestes, postures, attitudes corporelles) habituels ;

- Avec média technique non numérique (par exemple, par téléphone);

- Avec média numérique non spécifique (par exemple un moteur de recherche généraliste comme Google.fr);

- Avec média numérique spécifique (par exemple un moteur multi-utilisateur comme MUSE [Krishnappa, 2005], DESIRE, ou un système de navigation collaboratif comme Ariadne [Twidale et al, 1997])

(3) les phases de l'activité durant lesquelles la collaboration existe :

- Durant la phase pré-active, c'est-à-dire avant la recherche d'information effective (par exemple, lors de la planification, du choix des mots-clés, d'un brainstorming) ;

- Durant la phase active, c'est-à-dire pendant la recherche proprement dite (par exemple, en partageant les sites Web, par du conseil);

- Après la recherche (par exemple, lors de la mise en commun des résultats, lors de la synthèse et écriture finales).

Bien évidemment, les catégories d'activités ainsi définies ne sont pas exclusives les unes par rapport aux autres, et diverses combinaisons peuvent exister. Actuellement, l'une des typologies les plus utilisées est celle créée par [Twidale, Nichols \& Paice 1997]. Selon ces auteurs, les activités de recherche collaborative d'information peuvent être distinguées selon deux axes orthogonaux : un axe spatial permet de distinguer les activités selon que les collaborateurs sont proches physiquement ou non; un axe temporel distingue les activités selon que les individus collaborent de manière synchrone ou asynchrone. Ces deux axes sont des continuums le long desquels une situation particulière peut être positionnée et ainsi définie. D'ailleurs, pour chacune des quatre conditions possibles, les auteurs présentent des exemples (Figure 1). 


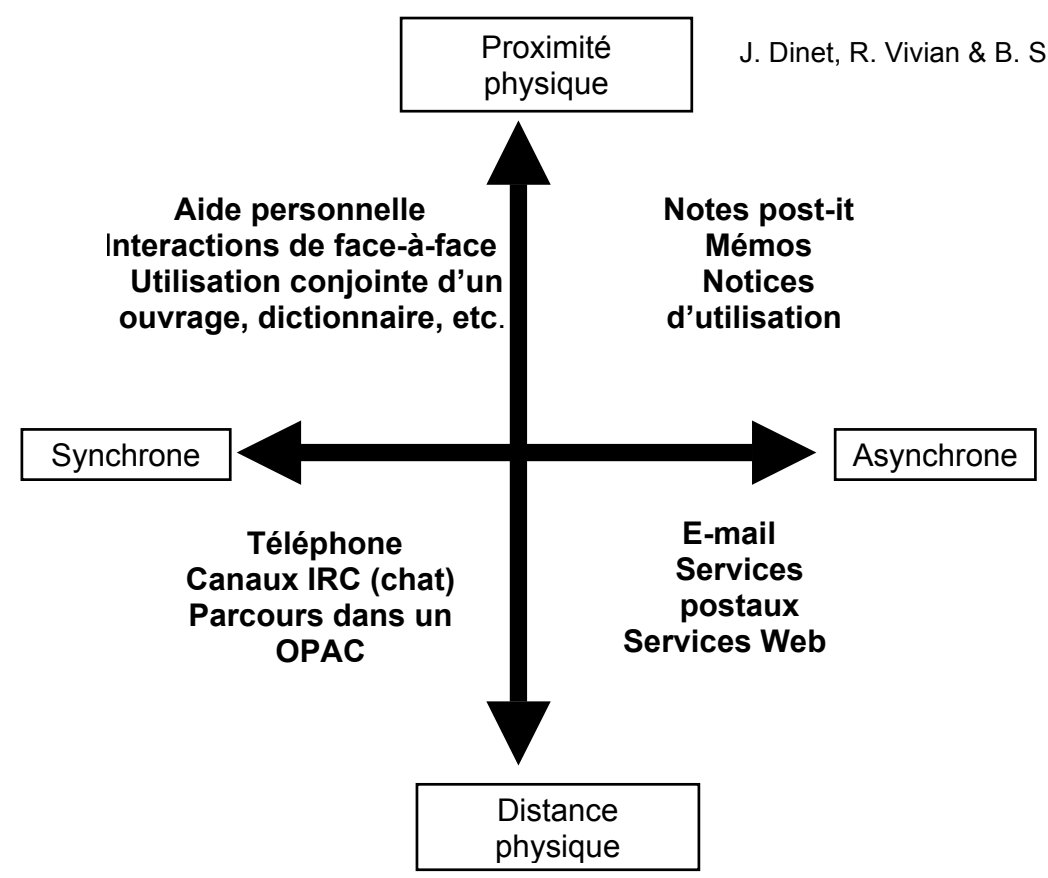

Figure 1. Typologie des activités de recherche collaborative d'information adaptée de Twidale, Nichols \& Paice [1997]

En matière de recherche collaborative d'information, les travaux peuvent être distingués en quatre grands groupes selon l'orientation théorique (et méthodologique) des auteurs, cette thématique étant interdisciplinaire (Foster, 2006) :

- Les sciences de l'information et de la communication (SIC) adoptent une approche multidimensionnelle dans laquelle une place privilégiée est donnée aux facteurs sociaux, liés à la situation. Ces derniers ont un impact sur les différentes phases associées directement ou indirectement à la recherche d'information, telles que la définition du besoin, l'acquisition d'information et son traitement, ou encore l'organisation de ces informations. Ainsi, sur la base de modèles initialement conçus pour rendre compte des situations de recherche d'information individuelle (tels que le modèle Information Search Process ou ISP de [Kuhlthau 1991]), de nouveaux modèles sont élaborés en insistant particulièrement sur les aspects sociaux et affectifs (par exemple, [Hyldegard 2006] [Hyldegard 2009] [Ingwersen \& Jarvelin 2005] [Karamuftuoglu 1998]). L'un des apports de ces approches est de mettre l'accent sur la dimension sociale, communicationnelle et affective liée à l'aspect collectif et collaboratif ;

- Le domaine des sciences et techniques (dont les mathématiques et l'informatique) expérimente depuis de nombreuses années des outils (logiciels, plates-formes, didacticiels, etc.) censés aider et faciliter le travail collaboratif dans sa totalité ou en partie. La phase de recherche collaborative d'information est alors parfois intégrée par le biais d'outils censés aider à la navigation de groupes d'utilisateurs dans des contenus hypertextuels [Dosser \& Perrimond 2001], permettre la production de requêtes plus pertinentes [Razen 2004], ou Journal d'Interaction Personne-Système, Vol. 2, Num. 1, Art.3, Janvier 2011 
encore faciliter le filtrage collaboratif [Denos, Berrut, Galllardo-Lopez \& Nguyen 2004]. Mais, rares sont les expériences ayant donné lieu à de réelles évaluations, qui plus est auprès de populations non expertes en informatique, et en situation réelle contextualisée. Malgré tout, ces études présentent l'avantage de tester dans certaines conditions l'utilisabilité de dispositifs techniques innovants ;

- La psychologie sociale et la psychologie cognitive proposent une approche anthropocentrée de la recherche collaborative d'information [Dinet, Chevalier \& Tricot sous presse]. L'objectif de ces sciences humaines est triple: d'une part, mieux comprendre les comportements réels et mécanismes mentaux sous-jacents impliqués lors de la recherche collaborative d'information; d'autre part, mieux cerner les difficultés et facteurs de résistance (voire d'échec) chez les individus; enfin, proposer des recommandations en termes d'aménagement ou de création de dispositifs pour faciliter l'activité. Ici, un dispositif dépasse largement un simple outil technique ou technologique et renvoie à toutes les dimensions qu'il faut nécessairement prendre en compte (état des connaissances de l'individu, facteurs temporels et spatiaux, contexte exact, consignes, etc.) ;

- Les approches pluridisciplinaires, telles que l'ergonomie ou l'intelligence artificielle, proposent d'aborder la recherche collaborative d'information à l'aide d'une part, de théories et méthodes issues de plusieurs courants antérieurs et d'autre part, de techniques et concepts nouvellement et spécifiquement créés (pour une synthèse, [Vivian \& Dinet 2009]).

En empruntant à chacune des approches précédemment décrites, depuis quelques années, est apparu un nouveau champ théorique appelé Collaborative Information Behaviour (CIB). (pour une synthèse, [Reddy \& Jansen 2008] [Hyldegard 2006] [Hyldegard 2009]) afin de mieux comprendre les comportements, les processus mentaux sous-jacents, les facteurs et les impacts de la dimension collaborative de la recherche d'information, particulièrement dans les environnements numériques en ligne sur l'internet.

\section{Recherche collaborative d'information et apprentissage}

Dans le cadre scolaire, des situations d'apprentissage de plus en plus courantes amènent les élèves à rechercher de l'information sur Internet, cette activité étant souvent menée par binômes pour des raisons pédagogiques ou à cause de contraintes matérielles. Pour Dillenbourg [1999], la collaboration et les interactions qu'elle entraîne (explications, désaccords ou régulations mutuelles) déclenchent des mécanismes cognitifs supplémentaires quant à la sélection des informations et leur transformation en connaissances, et peut réduire la charge cognitive. Selon lui, plusieurs conditions sont nécessaires pour que l'on puisse parler d'apprentissage collaboratif :

- Une situation collaborative, c'est-à-dire une tâche commune, doit exister ;

- Des interactions et échanges (notamment verbaux) entre les membres du groupe doivent se produire ;

- Des processus d'apprentissages collaboratifs doivent pouvoir être identifiés ;

- Enfin, il doit y avoir des effets de cette recherche collaborative tant pour le groupe que pour chaque individu constituant ce groupe.

Mais, si ces quatre conditions sont nécessaires, elles ne sont pas suffisantes [Dinet \& Tricot 2007]. Par exemple, le fait qu'une tâche commune existe ne suffit pas puisque rien n'indique qu'il y a similitude et homogénéité des représentations du but pour chaque collaborateur. D'ailleurs, de nombreux travaux ont montré qu'une même consigne pouvait 
donner lieu à de multiples représentations mentales selon les individus appartenant à un même groupe de travail (pour une synthèse, [Dinet \& Rouet 2002]). De même, rechercher ou faire rechercher des informations de manière collaborative nécessite des compétences de gestion de groupe et/ou de «management » que les jeunes usagers ne possèdent pas.

\section{Recherche individuelle versus recherche collaborative : facteurs de difficultés}

Quels liens entretiennent les activités de recherche individuelle d'information et les activités de recherche collaborative d'information? En d'autres termes, quelles compétences et facteurs de difficultés sont communs et/ou différents selon le contexte de réalisation de cette même activité (individuelle versus collaborative) ? Répondre à ces interrogations est d'autant plus crucial lorsqu'elles concernent de jeunes élèves car elles permettront de mieux concevoir notamment les scénarii pédagogiques.

Comme le faisait déjà remarquer Agosto [2002], les études qui se sont intéressées aux difficultés rencontrées par les jeunes utilisateurs lors de recherche d'information sont beaucoup moins nombreuses que celles qui se sont intéressées à ces difficultés chez les adultes. Néanmoins, certaines recherches ont examiné les comportements des jeunes utilisateurs et ont permis l'identification de domaines particulièrement problématiques pour cette population spécifique [Borgman, Hirsch, Walter, \& Gallagher 1999] [Chan, Tan, \& Wei 2000] [Hirsh 2000]. Cette identification des difficultés, notamment liées aux aspects psychologiques, est nécessaire si l'on souhaite mettre en place des séances susceptibles d'aider les jeunes usagers à réaliser plus efficacement leurs activités.

Que les recherches d'information soient réalisées individuellement ou de manière collaborative, les principales difficultés rencontrées par des jeunes élèves devant les réaliser sur Internet peuvent être regroupées dans l'une des catégories suivantes [Dinet, 2008] [Dinet \& Passerault 2009] :

- Les difficultés motrices : réaliser une recherche d'information sur Internet nécessite généralement d'utiliser un clavier alphanumérique et/ou une souris. Or, plusieurs études ont montré que les enfants des écoles primaires peuvent présenter de sérieuses difficultés dans la manipulation du clavier et de la souris [Erthal 1985] [Hooten 1989] [Solomon, 1993], ces difficultés pouvant perdurer pour certains jusqu'au collège [Borgman et al. 1999] [Dinet \& Passerault 2009]. De plus, cela pose la question de l'accessibilité des outils de recherche d'information via les claviers et les souris aux publics présentant des difficultés perceptives et/ou motrices avérées ;

- La faible motivation : comme pour la plupart des activités scolaires [Lieury \& Fenouillet 1996] [Tardif 1999], il semble exister une relation étroite entre le degré de motivation et la réussite dans une recherche d'information sur Internet [David et al., 2007] [Kuhlthau, Maniotes \& Caspari 2008] [Gross 1999; 2000]. En effet, certaines études ont cherché à démontrer que la faible motivation des élèves réalisant des recherches d'information sur Internet explique, en partie, leurs faibles performances (peu de références trouvées, passivité de l'élève durant l'activité, etc.). Le fait que le thème de la recherche soit généralement « externe » à l'élève (i.e., prescrit et défini par l'enseignant) serait responsable de cette faible implication des enfants dans l'activité ;

- Les connaissances déclaratives : les connaissances déclaratives sont liées aux mots, aux contenus, aux objectifs. Par exemple, plusieurs études ont montré que les termes produits 
par les enfants pour interroger les bases de données et/ou les moteurs de recherche comportent souvent des erreurs orthographiques [Borgman \& Siegfried 1992] [Hooten 1989] [Solomon 1993]. Lorsque le système documentaire répond qu'aucune référence n'a été trouvée et ce à cause d'une erreur orthographique, les jeunes utilisateurs considèrent volontiers que le «défaut» provient du système. De plus, pour un thème de recherche donné, les termes (ou mots-clefs) avec lesquels les enfants interrogent les bases documentaires sont d'une part, peu nombreux et d'autre part, peu variés [Moore \& St. George 1991] [Solomon 1993]. Enfin, les enfants ont des difficultés à déterminer quand ce qu'ils ont trouvé suffit pour répondre au besoin d'information, ils peuvent cesser de chercher pour d'autres raisons comme l'inconfort physique par exemple [Agosto 2002] ;

- Les connaissances procédurales : ce sont des connaissances qui sont de nature dynamique, implicites et concernent le «comment faire quelque chose » comme conduire une automobile, utiliser un logiciel, etc. Certaines difficultés des élèves sont directement liées à des «défauts 》 dans ces connaissances procédurales liées aux actions, méthodes et procédures. Ainsi, très logiquement, l'une des principales causes d'échec des élèves réalisant une recherche d'information sur Internet est le faible niveau de maîtrise de l'outil, même après une durée d'utilisation relativement importante [Dinet 2008] [Dinet \& Rouet 2002]. Toutefois, une recherche sur Internet implique avant tout une démarche intellectuelle, indépendamment de l'outil [Dinet 2008]. Or, rares sont les élèves de cycle élémentaire qui semblent connaître et maîtriser les procédures nécessaires à la réalisation d'une recherche d'information sur Internet. En effet, si les jeunes usagers semblent maîtriser de mieux en mieux les périphériques associés à l'activité de recherche d'information, il apparait qu'ils comprennent et maîtrisent très moyennement les flux d'information qui se passent « derrière l'écran ». Par exemple, il est difficile pour un jeune utilisateur de se construire une représentation mentale de ce qu'est Internet: le plus souvent, les enfants considèrent qu'Internet est simplement l'ordinateur [Dinet 2008]. La représentation d'Internet comme un espace n'ayant pas de réalité physique et indépendant de l'ordinateur arrive plus tardivement.

Dans le cas d'une recherche d'information menée de manière collaborative, il apparaît qu'un autre type de difficultés semble en partie expliquer les échecs des jeunes élèves : il s'agit des difficultés métacognitives. La métacognition est ici impliquée dans la gestion et supervision de l'activité, mais surtout dans la gestion des relations entre les membres réalisant l'activité. Malheureusement, les travaux sur l'implication des facteurs métacognitifs dans l'activité de recherche d'information sont apparus récemment [Dinet \& Rouet 2002]. D'une part, il apparaît que l'élève réalisant une recherche sur Internet anticipe peu ses activités, contrôle rarement ses actions et ajuste encore plus rarement ses comportements en cas de problèmes. D'autre part, il apparaît également que les jeunes élèves éprouvent des difficultés à gérer leur relation de travail et les activités au sein de leur groupe de travail (répartition des tâches, ajustement des conduites, etc.). Or, certaines recherches actuellement menées tendent à montrer que les échecs des jeunes élèves lors d'une recherche d'information sur Internet peuvent être dus à un défaut dans ces opérations d'anticipation, de contrôle et d'ajustement [Dinet, Chevalier \& Tricot sous presse], ces opérations se développant assez tardivement avec l'âge (11-13 ans) [Dinet \& Rouet 2002].

La gestion des relations sociales appartient au registre des compétences métacognitives. Plusieurs études tendent à montrer que les liens affectifs qui existent entre les collaborateurs 
devant rechercher ensemble des informations sur Internet et la gestion de ces liens affectifs influencent les comportements et performances [Crook 1998a ; 1998b] [Hyun 2005] [Vass 2002]. En effet, si les deux (ou plus) collaborateurs entretiennent des liens amicaux entre eux, la recherche d'information tend à être réalisée plus rapidement et plus efficacement. Sur la base de ces différentes études, un auteur prône même la recherche collaborative d'information en insistant sur le fait que « deux têtes cherchent mieux qu'une » [Lazonder 2005].

Pourtant, plusieurs remarques peuvent être émises à l'encontre de ces études, remarques qui justifient les recherches ici présentées :

- Les études qui montrent l'intérêt à prendre en compte les liens affectifs entre les collaborateurs lors d'une recherche d'information sur Internet ne s'intéressent généralement qu'à des usagers adultes (étudiants, voire experts d'un domaine) ;

- Les échantillons de participants sont très réduits : ces études, bien que très riches d'enseignements, sont plus des études de cas empiriques que des expérimentations.

Aussi, grâce à deux expérimentations présentées ci-après, nous avons tenté d'étudier les impacts réels du degré d'affinité entre les collaborateurs lorsqu'il s'agit de très jeunes usagers devant réaliser une recherche d'information collaborative sur Internet. L'un des principaux objectifs de la première étude est de quantifier et de qualifier l'impact de ce degré d'affinité sur les performances et comportements des jeunes utilisateurs en comparant les situations individuelles et collaboratives lors des recherches d'informations. L'un des principaux objectifs de la seconde étude est de se focaliser plus spécifiquement sur l'impact de ce même degré d'affinité sur les échanges verbaux entre élèves collaborant lors d'une recherche d'information sur Internet.

\section{ETUDE 1 : QUELS COMPORTEMENTS ?}

Dans cette première étude qui s'est déroulée de janvier à mai 2006, nous nous sommes intéressés aux comportements et stratégies de jeunes usagers (élèves de CM2) devant rechercher des informations sur Internet. Ces expérimentations ont été menées dans leur contexte scolaire habituel, afin de ne pas induire un quelconque effet lié à l'expérimentation en laboratoire. Trois conditions successives ont été testées : recherche seul (condition «SEUL »); en binôme avec un(e) autre élève avec le(la)quel(le) des liens amicaux sont entretenus (condition "AFFINITE + "); en binôme avec un(e) autre élève avec le(la)quel(le) aucun lien amical n'existe (condition « AFFINITE - »). Dans ces deux derniers cas, la collaboration des élèves était donc synchrone et explicite.

\section{Participants}

Dans cette étude, 52 élèves ( 24 garçons et 28 filles) scolarisés en CM2 ont participé (âge moyen $=9.4$ ans, écart-type $=0.6$ ans). Tous ces participants fréquentaient la même école, étaient issus d'un même bassin de recrutement géographique et tous étaient francophones. Aucun n'était redoublant et tous avaient l'habitude d'utiliser Internet pour rechercher des informations en moyenne $1 \mathrm{~h} 30$ par semaine à l'école. De plus, tous ont déclaré utiliser Internet chez eux depuis, en moyenne, 13 mois.

\section{Tâche et procédure}


Chaque élève devait rechercher des informations sur Internet afin de répondre à des questions relatives à l'histoire médiévale. Les consignes précisaient que l'objectif était de compléter les leçons (l'histoire médiévale étant abordée en cours) afin de préparer une exposition qui serait présentée aux parents en fin d'année scolaire.

Chaque élève devait rechercher des informations sur Internet relativement à l'histoire médiévale dans trois conditions successives, chaque session étant séparée par 15 jours :

- Session 1 : l'élève doit réaliser l'activité seul (condition « SEUL »);

- Session 2 : l'élève doit réaliser l'activité avec un(e) autre élève avec le(la)quel(le) des liens amicaux sont entretenus (condition «AFFINITE $+»)$;

- Session 3 : l'élève doit de nouveau réaliser l'activité avec un(e) autre élève, mais cette fois-ci, aucun lien amical n'existe entre les deux élèves (condition « AFFINITE - »).

Les trois sessions sont contrebalancées afin d'éviter que tous les participants ne voient les conditions dans le même ordre.

Ce sont les enseignants qui, forts de leurs expériences avec leurs élèves, ont permis de constituer les binômes pour la session 2 et la session 3. C'est d'ailleurs cette technique pour constituer les groupes d'élèves qui est utilisée dans la plupart des études s'intéressant aux comportements impliqués dans les activités collaboratives à l'école.

Les questions auxquelles les élèves devaient répondre étaient présentées dans un ordre contrebalancé entre les trois sessions pour éviter un éventuel effet d'ordre. Ainsi, il y avait six ordres différents dans lesquels les sessions étaient proposées : (session 1, puis session 2, puis session 3 ; session 3 , puis session 2, puis session 1 ; session 1 , puis session 3 , puis session 2 ; etc.). Pour chacune de ces trois sessions, ce sont 13 questions différentes liées à l'histoire médiévale auxquelles devaient répondre les élèves et/ou les binômes (Tableau 1). Puisque chaque élève et/ou binôme devait réaliser la recherche d'information sur Internet lors de trois sessions, chaque élève et/ou binôme devait donc répondre, en tout, à 39 questions. Les questions étaient présentées sur une feuille au format A4, des espaces suffisants étant laissés entre chaque question pour qu'ils puissent noter la(les) réponse(s). Le temps imparti pour réaliser l'activité était libre.

\begin{tabular}{|l|}
\hline Trouve 5 prénoms masculins du Moyen Age \\
\hline Trouve 5 prénoms féminins du Moyen Age \\
\hline Trouve une recette de cuisine du Moyen Age \\
\hline Trouve les noms de 3 rois du Moyen Age \\
\hline Trouve 3 instruments de musique du Moyen Age \\
\hline Trouve 5 armes du Moyen Age \\
\hline
\end{tabular}

Tableau 1. Exemples de questions auxquelles devaient répondre les élèves et/ou les binômes.

\section{Indicateurs comportementaux}

Pour chaque élève et/ou binôme, c'est-à-dire pour les trois conditions, plusieurs indicateurs ont été relevés, notamment en récupérant immédiatement après chaque session les historiques de leurs navigations :

- Le nombre de réponses données à la fin de l'activité (de 0 à 13) ; 
- Le pourcentage de bonnes réponses données ;

- Le nombre de pages Web explorées (une page a été considérée comme explorée si elle est restée affichée plus de 45 secondes ; ce choix de 45 secondes a été arbitrairement déterminé) ;

- Le nombre de cycle de recherche, un cycle correspondant à chaque validation d'une requête par les participants ;

- L'efficience, correspondant à un ratio calculé sur la base suivante : nombre de bonnes réponses données / nombre de pages Web consultées ;

- Le type de requêtes « incorrectes » produites, ces requêtes pouvant être incorrectes pour trois raisons :

(a) Elles peuvent comporter des erreurs orthographiques (par exemple, « életricité »);

(b) Elles peuvent ne pas correspondre à des mots effectifs (par exemple, lorsqu'un élève joue avec le clavier : « azertyuiiiooo »);

(c) Elles peuvent être hors sujet (i.e., non pertinentes), c'est-à-dire n'avoir aucun lien thématique avec le thème de la recherche (par exemple, un nom de chanteur ou chanteuse).

\section{Principaux résultats}

Pour chacune des trois conditions ( SEUL », «AFFINITE + », et «AFFINITE - »), les performances moyennes des élèves ont été relevées (Tableau 2).

Condition

\begin{tabular}{lccc}
\hline & Seul & Affinité - & Affinité + \\
\hline Nombre de bonnes réponses (sur 13) & 3.75 & 5.33 & 4.27 \\
& $(0.23)$ & $(0.65)$ & $(0.56)$ \\
Pourcentage de réponses correctes & 23.07 & 36.75 & 29.05 \\
& $(2.54)$ & $(2.32)$ & $(1.97)$ \\
Nombre de pages Web visitées & 26.81 & 24.05 & 24.77 \\
& $(2.12)$ & $(2.87)$ & $(3.01)$ \\
Efficience & 0.12 & 0.22 & 0.15 \\
& $(0.04)$ & $(0.06)$ & $(0.03)$ \\
Nombre de cycles de recherche & 19 & 16.33 & 15.44 \\
& $(2.5)$ & $(3.2)$ & $(3.9)$ \\
Requêtes ... & & & \\
$\ldots$ avec erreurs orthographiques & 19.44 & 13.7 & 14.09 \\
& $(1.4)$ & $(1.09)$ & $(0.98)$ \\
$\ldots$ non-mots & 5.46 & 5.29 & 6.29 \\
& $(0.87)$ & $(0.96)$ & $(0.78)$ \\
... non pertinentes & 1.4 & 0.2 & 3.87 \\
& $(0.02)$ & $(0.01)$ & $(0.06)$ \\
\hline
\end{tabular}

Tableau 2. Performances moyennes (et écart-types) pour les élèves, pour les trois conditions. 
Les analyses statistiques (ANOVAs) ont révélé plusieurs résultats intéressants :

- Les élèves recherchant individuellement les informations sur Internet (condition «SEUL ») trouvent significativement moins de bonnes réponses que ces mêmes élèves travaillant en binôme, quel que soit le degré d'affinité au sein de ce binôme $(\mathrm{p}<.0001)$;

- Les binômes trouvent significativement plus de bonnes réponses lorsque les élèves n'entretiennent pas de liens amicaux entre eux (différence entre «AFFINITE + » et «AFFINITE - ») $(\mathrm{p}<.001)$;

- Les élèves recherchant individuellement les informations sur Internet (condition «SEUL ») sont moins efficients que lorsqu'ils travaillent en binôme, quel que soit le degré d'affinité au sein de ce binôme ( $\mathrm{p}<.0001)$;

- Les binômes sont plus efficients lorsque les élèves n'entretiennent pas de liens amicaux entre eux («AFFINITE - $)(\mathrm{p}<.001)$.

En ce qui concerne les requêtes produites par les élèves (Tableau 2), nous pouvons remarquer que :

- Les élèves produisent significativement plus de requêtes comportant des erreurs orthographiques lorsqu'ils recherchent individuellement les informations sur Internet (condition « SEUL ») $(\mathrm{p}=.01)$;

- Les binômes produisent significativement plus de requêtes non pertinentes par rapport au thème de la recherche d'information si des liens amicaux existent entre les élèves $(«$ AFFINITE $+»)(p=.002)$.

En d'autres termes, cette première étude tend à montrer que si les élèves sont plus efficaces et plus efficients en situation de recherche collaborative d'information (lors de la navigation et lors de la production des requêtes), cette "plus-value» de la situation collaborative est surtout vraie lorsque les membres d'un binôme n'entretiennent aucun lien amical. En effet, lorsque les deux élèves membres d'un même binôme sont amis, d'une part, ils sont moins efficaces et moins efficients et d'autre part, ils produisent plus de requêtes non pertinentes par rapport au thème de la recherche.

Nous pouvons également noter que quel que soit l'ordre dans lesquels les élèves ont vu les trois sessions, la répétition de l'activité n'a eu aucun effet significatif sur les performances et comportements $(\mathrm{p}=.07)$.

Nous faisons l'hypothèse que les binômes constitués d'élèves qui entretiennent des liens amicaux entre eux sont moins efficaces et efficients que les binômes constitués d'élèves n'entretenant pas de tels liens à cause de digressions qui les conduiraient à une déconcentration et à une "perte» de temps. Aussi, pour tester cette hypothèse, est-il nécessaire d'analyser ce qui se passe au sein des binômes, en termes d'interactions notamment verbales.

\section{4. ÉTUDE 2 : QUELLES INTERACTIONS?}

Dans cette seconde étude qui s'est déroulée d'avril à juin 2007, nous nous sommes particulièrement intéressés aux interactions verbales entre les deux membres des binômes devant rechercher, ensemble, des informations sur Internet. 


\section{Participants}

Dans cette étude, 24 élèves de sexe féminin, scolarisées en CM2 ont participé (âge moyen = 8.7 ans, écart-type $=0.7$ ans). Toutes ces participantes fréquentaient la même école, étaient issues d'un même bassin de recrutement géographique et toutes étaient francophones. Aucune n'était redoublante et toutes avaient l'habitude d'utiliser Internet pour rechercher des informations en moyenne $1 \mathrm{~h} 30$ par semaine à l'école. De plus, toutes ont déclaré utiliser Internet chez elles depuis, en moyenne, 11.8 mois. Tous les participants étaient de sexe féminin car une étude conduite juste avant cette seconde expérience tend à montrer que la composition des binômes selon le genre a une influence sur les comportements [Hyldegard 2009].

\section{Tâche et procédure}

La tâche et la procédure étaient identiques à celles utilisées lors de la première étude à une différence près : nous intéressant aux interactions verbales, la condition «SEUL 》 a été supprimée. Chacune des élèves devait donc rechercher des informations sur Internet relativement à l'histoire médiévale dans deux conditions successives, chaque condition étant séparée par 15 jours :

- Session 1 : l'élève doit réaliser l'activité avec un(e) autre élève avec le(la)quel(le) des liens amicaux sont entretenus (condition « AFFINITE $+»)$;

- Session 2 : l'élève doit de nouveau réaliser l'activité avec un(e) autre élève, mais cette fois-ci, aucun lien amical n'existe entre les deux élèves (condition «AFFINITE - »)

Les deux conditions étaient contrebalancées pour les participantes. Enfin, les matériels (questions, ordinateurs, moteurs de recherche, etc.) et les consignes étaient identiques à la première étude.

Toutes les séances de recherche collaborative d'information ont été enregistrées audiovisuellement. Ces enregistrements ont été par la suite dépouillés grâce à l'emploi d'une grille d'analyse des interactions verbales standardisée et déjà validée [Hmelo-Silver 2003]. Cette grille permet, sur la base des tours de paroles, de distinguer quatre grands types d'interactions verbales existant lors d'une recherche collaborative d'information :

1. Les interactions correspondant à un conflit, ce conflit concernant généralement le thème ou la procédure (« tu fais n'importe quoi là ! »; « t'as rien compris ! »; « je veux plus jamais travailler avec toi !»);

2. Les interactions relevant de questionnements que l'un des collaborateurs adresse à l'autre, ces questionnements pouvant concerner le but, la démarche, les concepts, etc. («t'as compris ce que le maître veut ? »; « tu te souviens de comment il faut faire ?»);

3. Les interactions correspondant aux réponses que le collaborateur adresse en réponse au questionnement précédemment évoqué ;

4. Les interactions visant à faciliter le travail collaboratif, soit par des encouragements (« ha oui, c'est comme ça, t'as raison!»), soit par des auto-congratulations (« t'as vu ça ? J'ai trouvé du premier coup !»).

Si la grille utilisée permet une analyse très fine des interactions verbales [Hmelo-Silver 2003], nous ne présentons ici que les résultats correspondant aux quatre grands types d'interactions explicités ci-dessus.

\section{Indicateurs comportementaux}


Pour chaque binôme, au vu des résultats de la première étude, seuls les indicateurs suivants ont été relevés :

- Le nombre de réponses données à la fin de l'activité (de 0 à 13) ;

- Le pourcentage de bonnes réponses données ;

- La distribution des tours de paroles (en pourcentage) pour chacun des quatre grands types d'interaction : conflit ; questionnement ; réponse ; facilitation.

\section{Principaux résultats}

Comme le montre le Tableau 3 et comme le confirment les analyses statistiques (ANOVAs), les résultats obtenus dans cette seconde étude sont similaires à ceux obtenus dans la première étude pour nos deux premiers indicateurs comportementaux (le nombre de réponses données à la fin de l'activité et le pourcentage de bonnes réponses). De même, comme cela a été constaté pour la première étude, la répétition de l'activité n'a eu aucun effet significatif sur les performances et comportements $(\mathrm{p}=.081)$.

\begin{tabular}{lcc}
\hline & Affinité - & Affinité + \\
\hline Nombre de bonnes réponses (sur 13) & 5.17 & 4.48 \\
& $(0.57)$ & $(0.49)$ \\
Pourcentage de réponses correctes & 41.38 & 30.15 \\
& $(1.78)$ & $(0.99)$ \\
\hline
\end{tabular}

Tableau 3. Performances moyennes (et écart-types) pour les élèves, pour les deux conditions.

Dans cette seconde expérience, tout comme dans la première, les binômes trouvent significativement plus de bonnes réponses lorsque les élèves n'entretiennent pas de liens amicaux entre eux ( $«$ AFFINITE $-»)(\mathrm{p}<.001)$.

En ce qui concerne les interactions verbales au sein des binômes (Tableau 4), deux résultats majeurs ont été obtenus :

- Lorsque les élèves recherchent des informations sur Internet avec une amie (" AFFINITE $+»)$, il y a beaucoup plus d'interactions reflétant des conflits entre les deux collaboratrices $(\mathrm{p}=.002)$;

- Lorsque les élèves recherchent des informations avec une autre élève avec laquelle aucun lien amical n'existe a priori (« AFFINITE - »), il y a significativement plus d'interactions facilitatrices qui sont produites $(\mathrm{p}<.001)$. Aucun autre résultat significatif n'a été relevé. 


\begin{tabular}{lcc}
\hline & Affinité - & Affinité + \\
\hline CONFLIT & 10.9 & 15.7 \\
& $(0.67)$ & $(1.73)$ \\
QUESTIONNEMENT & 31.9 & 37.2 \\
& $(3.98)$ & $(4.02)$ \\
REPONSES & 39.9 & 41.9 \\
& $(3.87)$ & $(4.5)$ \\
FACILITATION & 15.1 & 8.2 \\
& $(2.13)$ & $(1.14)$ \\
\hline
\end{tabular}

Tableau 4. Distribution des tours de parole (en pourcentage) selon leur nature.

\section{DISCUSSION GENERALE ET PERSPECTIVES}

Nos deux expérimentations tendent donc à confirmer des résultats d'études antérieures [Crook 1998] [Crook 1998b] [Hyun 2005] [Vass 2002] puisqu'il apparaît que des élèves travaillant en binômes trouvent effectivement plus de bonnes réponses (i.e., informations pertinentes) que lorsque ces mêmes élèves travaillent seuls.

En plus de confirmer les résultats d'études antérieures, nos expérimentations les complètent en démontrant que le degré d'affinité qu'entretiennent deux élèves membres d'un même binôme conditionne en grande partie l'efficacité et les performances du binôme : - D'une part, les binômes trouvent significativement plus de bonnes réponses (i.e., informations pertinentes) lorsque les élèves n'entretiennent pas de lien amical entre eux ;

- D'autre part, les binômes produisent significativement plus de requêtes non pertinentes par rapport au thème de la recherche d'information si des liens amicaux existent entre les élèves ;

- Enfin, les interactions verbales d'élèves entretenant des liens amicaux sont davantage orientées vers des conflits tandis que les interactions verbales visent plus à faciliter le travail du binôme dans le cas où les deux élèves n'entretiennent pas de lien amical entre eux.

La recherche collaborative d'information sur Internet semble donc présenter des avantages pour de jeunes usagers (scolarisés en CM2) : d'une part, en ce qui concerne la sélection des informations, le travail en binôme améliore le nombre des informations pertinentes trouvées. D'autre part, en ce qui concerne la phase d'interrogation documentaire (i.e., production des requêtes), le travail collaboratif entraîne un nombre moindre d'erreurs orthographiques, comme si « l'autre » jouait le rôle de superviseur et de correcteur.

En revanche, ces avantages liés à la situation collaborative lors d'une recherche d'information sur Internet semblent très atténués, voire annulés, si les élèves membres d'un binôme devant réaliser ensemble l'activité entretiennent des liens amicaux. En effet, lorsque ce sont deux amis qui collaborent à rechercher les informations sur Internet, les performances diminuent (par exemple : diminution du nombre d'informations pertinentes trouvées, augmentation du nombre de requêtes non pertinentes) et leurs interactions verbales sont surtout centrées sur des conflits et des règlements de conflits. À l'inverse, lorsque les binômes sont constitués par deux élèves n'entretenant pas de lien amical particulier, les performances augmentent et les interactions verbales sont surtout centrées sur la tâche et sa facilitation. 
Plusieurs biais méthodologiques existent dans nos deux expérimentations. L'un des principaux concerne l'opérationnalisation de l'amitié (i.e., le degré d'affinité) afin de constituer les binômes. En effet, dans notre étude comme dans la plupart des travaux antérieurs, la constitution des binômes selon le degré d'affinité entre les élèves a été opérée par les enseignants. Les deux arguments majeurs à l'emploi de cette méthode sont les suivants : d'une part, les enseignants sont ceux qui connaissent le mieux les relations qu'entretiennent leurs élèves, et l'expérimentation s'appuie sur la manière dont ils déterminent les affinités dans la situation en classe ; d'autre part, c'est la méthode la moins coûteuse pour constituer les groupes. Or, il est raisonnable de penser qu'avoir recours à un indicateur plus objectif serait tout à fait pertinent. Par exemple, dans une étude future, il est envisagé d'utiliser l'une des méthodes actuellement développées dans les études récentes qui s'intéressent à l'impact de l'amitié sur les relations entre les jeunes apprenants (pour une synthèse, [Hanham \& McCormick 2009]).

Néanmoins, malgré ces biais, les résultats que nous avons obtenus amènent à s'interroger sur plusieurs points :

- Au niveau théorique, il convient de réfléchir à la manière dont on peut inclure les aspects affectifs dans les modèles récents censés rendre compte des comportements et processus mentaux impliqués lors de recherches collaboratives [Dinet \& Tricot 2007] [Dinet, Chevalier \& Tricot sous presse]. En effet, si certains modèles insistent sur la nécessité de prendre en compte les dimensions liées aux émotions et aux affects dans les activités de recherche d'information, nous manquons encore cruellement de données quantitatives quant aux impacts de ces dimensions ;

- Au niveau pédagogique, il est nécessaire de s'interroger sur les facteurs et dimensions qu'il faudrait et/ou serait possible de prendre en compte pour constituer des groupes de travail [Dinet 2007];

- Au niveau ergonomique, il est primordial de réfléchir à des systèmes et/ou dispositifs pouvant aider les jeunes usagers à réaliser l'activité de recherche d'information de manière collaborative. À ce propos, un nouvel outil a été développé à l'Université Paul Verlaine Metz, cet outil permettant de mieux gérer les activités des groupes de «chercheurs d'informations ", d'une part par les membres du groupe eux-mêmes et d'autre part, par un éventuel tuteur ou enseignant qui supervise l'activité [Vivian \& Dinet 2009].

\section{REMERCIEMENTS}

Nous tenons à remercier ici tous les élèves, enseignants, parents d'élèves et directeurs d'école sans lesquels ces études n'auraient pas pu être réalisées.

\section{RÉFÉRENCES}

Agosto, D. 2002. Bounded Rationality and Satisficing in Young People's Web-Based Decision Making. Journal of the American Society for Information Science and Technology, 53 (1), 16-27

Bharat, K. 2000. SearchPad: explicit capture of search context to support web search. Computer Networks, 33, 493-501Borgman, C., \& Siegfried, S. L. 1992. Getty's 
synoname $^{\mathrm{TM}}$ and its cousins: a survey of applications of personal name matching algorithms. Journal of the American Society for Information Science, 43(7), 459-476.

Borgman, C., Hirsch, S. G., Walter, V. A., Gallagher, A. L. 1999. Children's searching behavior on browsing and keyword online catalogs: the science library catalog project. Journal of the American Society for Information Science, 46(9), 663-684.

Chan, H. C., Tan, B. C. Y., \& Wei, K. K. 2000. Three important determinants of user performance for database retrieval. International Journal of Human-Computer Studies, 51, 895-918.

Cockburn, A. \& McKenzie, B. 2001. What do web users do? An empirical analysis of web use. International Journal of Human-Computer Studies, 54, 903-922.

Crook, C. 1998a. Children as computer users: The case of collaborative learning. Computers \& Education, 30(3), 237-247.

Crook, C. 1998b. Computers in the community of class-rooms. In: Littleton, K. and Light, P., Editors (pp.103-117), Learning with computers: Analysing productive interaction, Routledge, London.

David, P., Song, M., Hayes, A., \& Fredin, E. S. (2007). A cyclic model of information seeking in hyperlinked environments: The role of goals, self-efficacy, and intrinsic motivation. International Journal of Human-Computer Studies, 65, 170-182.

deVries, B., van der Meij, H., \& Lazonder, A. W. (2008). Supporting reflective web searching in elementary schools. Computers in Human Behavior, 24, 649-665

Denos, N., Berrut, C., Gallardo-Lopez, L., \& Nguyen, A.-T. 2004. COCofil : une plateforme de filtrage collaboratif orientée vers la communauté. Actes de CORIA'2004, (pp. 9-2), 10-12 septembre 2004, Toulouse.

Diamadis, E.T. \& Polyzos, G.C. 2004. Efficient cooperative searching on the Web: system design and evaluation. International Journal of Human-Computer Studies, 61, 699-724.

Dillenbourg, P. 1999. What do you mean by collaborative learning ? In P. Dillenbourg (Ed.), Collaborative-learning: Cognitive and Computational Approaches. Oxford, G.B., Elsevier, p. 1-19.

Dinet, J. (dir) 2008. Usages, usagers et compétences informationnelles au 21e siècle, Paris, Hermès Science Lavoisier.

Dinet, J. 2007. Deux têtes cherchent mieux qu'une ? Oui, mais ... Médialog, 63, 38-41.

Dinet, J. \& Passerault, J.-M 2009. La recherche documentaire informatisée par les élèves : pour une approche centrée - usager. Revue Hermès, Paris, CNRS.

Dinet, J. \& Rouet, J.-F. 2002. La recherche d'information : processus cognitifs, facteurs de difficultés et dimension de l'expertise. In C. Paganelli (Ed.), Interaction homme-machine et recherche d'information (pp.133-161). Paris : Hermès.

Dinet, J. \& Tricot, A. 2007. Recherche d'information dans les documents électroniques. In A. Chevalier \& A. Tricot (Eds.), Ergonomie cognitive des documents électroniques. Paris : Presses Universitaires de France.

Dinet, J. Chevalier, A. \& Tricot, A. (sous presse). Information searching: A review. European Review of Applied Psychology.

Dinet, J., Simonnot, B., \& Vivian, R. 2008. La recherche collaborative d'information sur Internet: impact du lien affectif antre les jeunes collaborateurs. Actes de la 20ème conférence sur l'Interaction Homme - Machine IHM'2008 (pp.137-144), 2-5 septembre 2008, Metz.

Dosser, F. \& Perrimond, L. 2001. Aide à la navigation sur le Web pour un groupe d'utilisateurs. Internship report, ESSI and INRIA project, 25 pages, April 2001. 
Dumais, S., Cutrell, E., Chen, H. 2001. Optimizing search by showing results in context. In CHI'01, Proceedings of the ACM Conference on Human-Computer Interaction (March 31 - April 5, 2001), Seattle, USA, ACM Press, New-York, 277-284.

Erthal, M. J. 1985. The status of keyboarding. The Journal of Business Education, 60, 192193.

Fidel, R., Pejtersen, A. M., Cleal, B., \& Bruce, H. (2004). A Multidimensional Approach to the Study of Human-Information Interaction: A Case Study of Collaborative Information Retrieval. Journal of the Amercian Society for Information Science andTechnology, 55 (11), 939-953.

Foster, J. 2006. Collaborative Information Seeking and Retrieval. Annual Review of Information Science and Technology, 40, 329-356

Gross, M. 1999. Imposed queries in the school library media center: a descriptive study. Library and Information Science Research, 21, 501-521.

Gross, M. 2000. Imposed and self-generated query in the elementary school environment. In R. M. Branch, M. A. Fitgerald (dir.), Educational media and technolgy yearbook 2000, Englewood, CO, Libraries Unlimited, p.120-129.

Hanham, J., \& McCormick, J. 2009. Group work in schools with close friends and acquaintances: Linking self-processes with group processes. Learning and Instruction, 19 (3), 214-227.

Hansen, P. \& Jarvelin, K. 2005. Collaborative Information Retrieval in an informationintensive domain. Information Processing \& Management, 41(5), 1101-1119.

Hmelo-Silver, 2003. C.E. Analyzing collaborative knowledge construction: Multiple methods for integrated understanding. Computers \& Education, 41(4), 397-420.

Hirsch, S. G. 2000. Children's relevance criteria and information seeking on electronic resources. Journal of the American Society for Information Science, 50(14), 1265-1283.

Hooten, P. A. 1989. Online catalogs: will they improve children's access ? Journal of Youth Services in Libraries, 2, 267-272.

Hyldegård, J. 2006. Collaborative information behaviour: exploring Kuhlthau's Information Search Process model in a group-based educational setting. Information Processing \& Management, 42 (1), 276-298.

Hyldegård, J. 2009. Beyond the search process - Exploring group members' information behavior in context. Information Processing \& Management, 45, 142-158.

Hyun, E. 2005. A study of 5- to 6-year-old children' peer dynamics and dialectical learning in a computer-based technology-rich classroom environment. Computers \& Education, 44 (1), 69-91.

Karamuftuoglu, M. 1998.Collaborative Information Retrieval: Toward a social informatics view of IR interaction. Journal of the American Society for Information Science, 49 (12), 1070-1080.

Krishnappa, R. 2005. Multi-user search engine (muse): Supporting collaborative information seeking and retrieval. Master's Thesis, University of Missouri - Rolla, Rolla, USA.

Kuhlthau, C. C. 1991. Inside the search process: seeking meaning from the users perspective. Journal of the American Society for Information Science, 42, (5), 361-371.

Kuhlthau, C. C., Maniotes, L.K., \& Caspari, A.K. 2008. Guided Inquiry: Learning in the 21st Century, New-York, Greenwood Publishing Group. 
Lazonder, A. 2005. Do two heads search better than one? Effects of student collaboration on web search be-haviour and search outcomes. British Journal of Educational Technology, 36(3), 465-475.

Lieury, A., \& Fenouillet, F. 1996. Motivation et réussite scolaire. Paris, Éditions Dunod.

Moore, P. A., St. George, A. 1991. Children as information seekers: the cognitive demands of books and library systems. School Library Media Quaterly, 19, 161-168.

Reddy, M. \& Jansen, B.J. 2008. A model for understanding collaborative information behavior in context: A study of two healthcare teams. Information Processing \& Management, 44 (1), 231-256.

Solomon, P. 1993. Children's information retrieval behavior: a case analysis of an OPAC. Journal of the American Society for Information Science, 44, 245-264.

Tardif, J. 1999. Pour un enseignement stratégique : l'apport de la psychologie cognitive. Montréal, Éditions Logique.

Twidale, M.B., Nichols, D.M., \& Paice, C.D. 1997. Browsing is a collaborative process. Information Processing \& Management, 33 (6), 761-783.

Vass, E. 2002. Friendship and collaborative creative writing in the primary classroom. Journal of Computer Assisted Learning, 18(1), 102-110.

Vivian, R., \& Dinet, J. 2009. RCI WEB : Présentation des premiers résultats de l'utilisation d'un système collaboratif de recherche d'information centré utilisateur. RIHM, 9(2), 85110 .

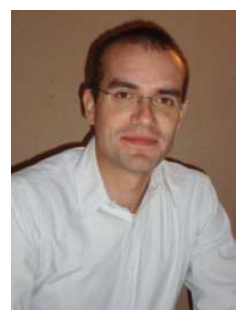

Jérôme Dinet est maître de conférences en Psychologie et Ergonomie cognitives à l'Université Paul Verlaine - Metz. Il est directeur du département de Psychologie, responsable du Master «Psychologie du travail », et membre du laboratoire INTERPSY-ETIC. Ses travaux de recherche portent essentiellement sur les comportements et processus cognitifs impliqués lors des activités de recherche d'informations. Il est également directeur de collection chez Hermès Sciences Publishing et relecteur pour plusieurs revues internationales telles que "Behaviour and Information Technology » et « Journal of Computer Assisted Learning ». Il est aussi expert - évaluateur auprès d'organismes tels que le Conseil de Recherches en Sciences Naturelles et en Génie du Canada ou encore l'Agence Nationale de la Recherche (ANR). Adresse électronique: dinet@univ-metz.fr

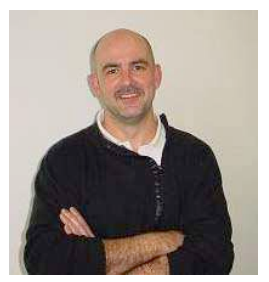

Robin Vivian est maître de conférences en informatique à l'université Paul Verlaine - Metz depuis septembre 1993. Il s'intéresse aux interactions avec les terminaux nomades (Projet ECIM de la ville de Metz), à l'analyse des comportements lors des enseignements en ligne (Projet UNSA éducation) et plus généralement les processus d'interaction avec les nouvelles technologies de l'information (Projet CVCE). Il étudie aussi les comportements et processus cognitifs des individus interagissant avec des environnements informationnels utilisant les NTIC (Internet, site web, etc.) dans des situations individuelles ou collaboratives. Il est le correspondant d' INTERPSY- 
ETIC du groupe de recherche CNRS «psychologie et ergonomique ». Adresse électronique : vivian@univ-metz.fr

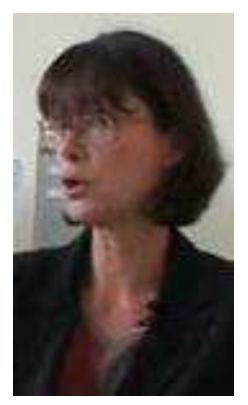

Brigitte Simonnot est maître de conférences en Sciences de l'information et de la Communication à l'Université Paul VerlaineMetz. Elle est membre du Centre de recherche sur les Médiations (EA 3476). Ses travaux de recherche portent essentiellement sur les usages des technologies de l'information et de la communication, et sur l'analyse des dispositifs d'accès à l'information, notamment les moteurs de recherche. Le domaine de la formation est un secteur d'application privilégié de ses travaux. Elle est membre du comité de rédaction de la revue Questions de Communication, et relectrice pour des conférences comme IHM ou des revues comme Etudes de communication. Adresse électronique : simonnot@univ-metz.fr 